\title{
Holi colors and chemical contact keratitis
}

Eye (2018) 32, 1-3; doi:10.1038/eye.2017.223; published online 20 October 2017

Having originated in India and celebrated across the world, 'Holi' marks the onset of spring. ${ }^{1}$ It symbolises the surrender of lust, forgiving the past mishaps and embracing each other, by immersing them in vibrant colors. Throwing of colours on each other is the hallmark of this festival. However, the enthusiasm of this festival may be marred by its alarming health consequences.

Traditionally, holi colors were derived from natural sources and are either particulate powders or liquid splashes, applied by hand, toy guns, or pounding balloons. But off-late, these hues have been contaminated with hazardous compounds such as malachite green, rhodamine, gentian violet and auramine $\mathrm{O}$, proven by High performance liquid chromatography., ${ }^{2,3}$ Other common adulterants include lead oxide, copper sulphate, mercury sulphite, chromium iodide, silica, mica dust etc. to impart attractive colours to carrier substances. Even fungal contamination of such powders has been proven. ${ }^{4}$ These powders, through contact with skin (dermatosis), respiratory tract or eyes may provoke unsought complications. ${ }^{5,6}$ Being poorly biodegradable and resistant to the conventional waste water purification processes, they are harmful to the environment too. ${ }^{7}$

With respect to the eye, the chemicals, their constituent endotoxins and fungal contamination produce ocular surface toxicity by induction of oxidative bursts in granulocytes. ${ }^{4}$ The ocular manifestations may vary from a mild chemical conjunctivitis to a secondarily infected corneal ulcer, all falling under the spectrum of ocular alkaline injury. ${ }^{5}$ However little is known about the specific clinical characteristics induced by these colours.

We studied 13 consecutive patients soon after the holi festival, who presented with ocular complaints to our out-patient or casualty services. The mean age of the sample population was $33 \pm 10.49$ years. All of them complained of foreign body sensation, 11/13 had ocular pain, $6 / 13$ showed signs of redness and excessive watering. Their presenting vision ranged from $20 / 40$ to $20 / 125$ (ETDRS). On fluorescein staining, 10/13 eyes demonstrated punctate keratopathy and 3/13 had a frank epithelial defect (Figure 1a). In cases of keratopathy, heaped up epithelium in a linear meandering fashion was observed, similar to a 'wave-like epitheliopathy', in the paracentral area, best appreciated under cobalt blue filter after fluorescein staining (Figure 1b). They were managed with copious saline irrigation followed by prophylactic topical antibiotics, lubricants and low potency steroids. The epitheliopathy took 3 weeks on an average to resolve; and also delayed epithelial healing was seen in patients with epithelial defects. Cases of similar epitheliopathy have been described in literature due to topical glaucoma medications, contact lens usage and their solutions, previous ocular surgeries and systemic inflammatory disorders, ${ }^{8}$ either arising from the limbus or from the site of corneal incisions. Confocal microscopy revealed abnormal basal epithelial cells in the former and basal nerve plexus damage in the latter.,, 10

In our series, the epitheliopathy might have been due to the direct toxic effect of the chemicals or local inflammatory cytokines induced by the holi powder constituents. ${ }^{4}$ On confocal microscopy (Confoscan 4, Nidek technologies, Tokyo, Japan), we found abnormal extraneous deposits in the layers involving the corneal epithelium and stroma. The amount of deposition decreased from epithelium to the deep stroma, corresponding with the depth of diffusion of the toxins, with the maximum being in epithelium (Figures 1c-e). It was accompanied with keratocyte loss (Figure 1f) and an increase in inflammatory reaction with generalised haze depicting stromal edema. We observed that the chemical injury caused by holi colors did not fit into the conventional Roper Hall classification and that, a separate system of classification is 

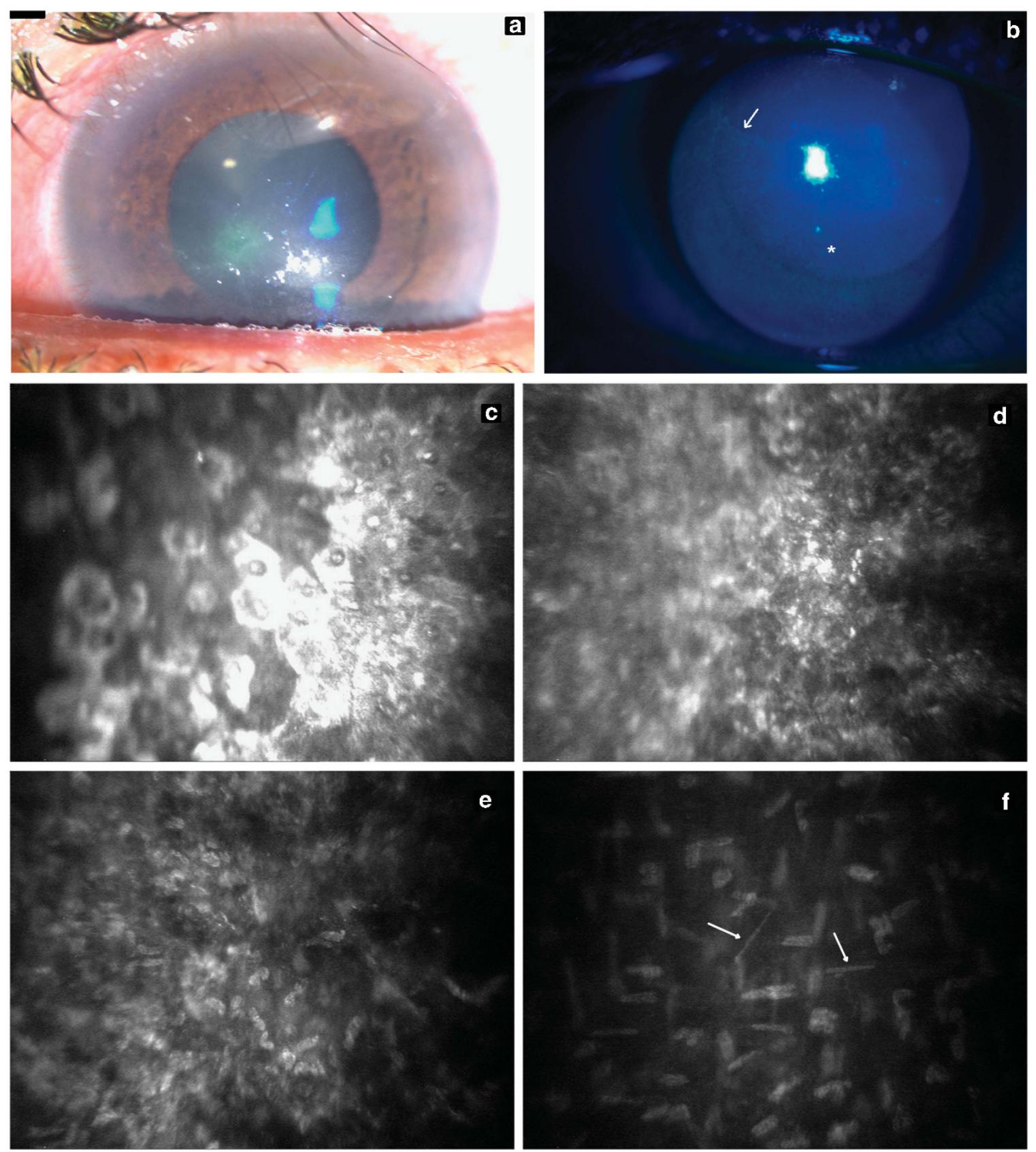

Figure 1 (a) Slit lamp biomicroscopic photograph shows corneal edema and an irregular epithelial defect of $2 * 1.5 \mathrm{~mm}$ at the centre. (b) Examination under cobalt blue filter after fluorescein staining shows linear heaped up epithelium in a waveform pattern in the paracentral area (white arrow). Surrounding punctate staining is also visible (white *). (c) Confocal microscopy image sectioning the superficial corneal epithelium showing hyperreflective extraneous deposits. (d) Confocal microscopy image sectioning the deep epithelium showing the same as previous image, but with decreased intensity. (e) Confocal microscopy image sectioning the superficial stroma which also shows the presence of deposits. (f) Confocal microscopy image sectioning the deep stroma which does not show any deposits, but demonstrates keratocyte apoptosis (white arrows).

required to grade the extent of injury in the absence of limbal stem ischemia. Owing to their different morphological structure, the conjuctival and the limbal stem cells were found to have a higher stress threshold to these agents in our series.
Very few marketed colour packets have a warning to avoid contact with skin or mucosa, ${ }^{4}$ as the manufacturing small scale industries fall short of directions and scrutiny. The low cost of these adulterated colors and essence during festive season ease their rampant availability. 
Despite being a widespread menace in Northern India, the hazards produced by these holi colors is not recognised, taught or highlighted anywhere in literature, including the textbooks and journals. Unfortunately, poor awareness and callousness exists regarding the dangers produced by these adulterated compounds, and no steps have been taken in India to counter this issue. US-FDA has on the other hand proposed malachite green, a frequent constituent of holi colors, as a priority agent for carcinogenicity tests. ${ }^{3}$ It is not difficult to fathom that these very toxins are a major health peril for the underprivileged population employed in its manufacture. In order to preserve the essence of the festival, these toxins need to be urgently substituted with natural colors. Government imposed bans on prohibition of manufacturing, sale and use of these chemicals are mandatory. It is also high time to provoke awareness in the community, by public health education programmes, underscoring the avoidable adverse health hazards. Recommendation guidelines regarding personal safety measures and first aid for these injuries by the national health experts is another basic unmet need.

Ocular injuries during holi primarily involve the young population, ${ }^{5}$ and so, have an adverse effect on the disability adjusted life years and the nation's productivity. Primary prevention is always better than cure. A few precautions for personal safety include the application of coconut oil over the skin and hair, which can act as a possible shield, sunglasses, and evading the use of contact lenses during such activities. The mentioned hazards can again be circumvented altogether by alternative ways of celebrations involving natural colours. ${ }^{11}$ It's time for realization and change while the essence of the festival remains.

\section{Conflict of interest}

The authors declare no conflict of interest.

\section{Acknowledgements}

We would like to acknowledge Mrs Meena Verma BSc (Optom) for aiding in the analysis of confocal miscroscopy images.

\section{References}

1 Holi festivals in Britain. The Telegraph [Internet]. 2016 Feb 4 [cited 2017 Jul 7]; Available from http:/ /www.telegraph.co. $\mathrm{uk} /$ travel/destinations/europe/united-kingdom/articles/ Holi-festivals-in-Britain/.

2 Ocular Chemical Injuries: Chemical Characterizations and Clinical Profile Correlations. ResearchGate. [cited 2017 Jul 7]. Available from https:/ / www.researchgate.net/publication/ 265047278_Ocular_Chemical_Injuries_Chemical_ Characterizations_and_Clinical_Profile_Correlations.

3 Velpandian T, Saha K, Ravi AK, Kumari SS, Biswas NR, Ghose S. Ocular hazards of the colors used during the festival-of-colors (Holi) in India-malachite green toxicity. J Hazard Mater. 2007; 139(2): 204-208.

4 Bossmann K, Bach S, Höflich C, Valtanen K, Heinze R, Neumann A et al. Holi colours contain PM10 and can induce pro-inflammatory responses. J Occup Med Toxicol 2016; 11: 42.

5 Dada T, Sharma N, Kumar A. Chemical injury due to colours used at the festival of Holi. Natl Med J India. 1997; 10 (5): 256.

6 Ghosh SK, Bandyopadhyay D, Chatterjee G, Saha D. The 'Holi' dermatoses: Annual spate of skin diseases following the spring festival in India. Indian J Dermatol 2009; 54(3): 240.

7 Impact of 'Holi' on the environment: A scientific study. ResearchGate. [cited 2017 Jul 7]. Available from https:/ / www.researchgate.net/publication/229066703_Impact_of_ 'Holi'_on_the_environment_A_scientific_study.

8 D'Aversa G, Luchs JL, Fox MJ, Rosenbaum PS, Udell IJ. Advancing wave-like epitheliopathy. Clinical features and treatment. Ophthalmology 1997; 104(6): 962-969.

9 Huang Y-H, Chu H-S, Hu F-R, Wang I-J, Hou Y-C, Chen W-L. Recurrent advancing wavelike epitheliopathy from the opposite side of the initial presentation. Cornea 2008; 27(1): 111-113.

10 Babu K, Narasimha Murthy K, Ramachandra Murthy K. Wavelike epitheliopathy after phacoemulsification: role of in vivo confocal microscopy. Cornea 2007; 26(6): 747-748.

11 Science behind Holi, the festival of Colours [Internet]. [cited 2017 Jul 7]. Available from http://ncsm.gov.in/ $? \mathrm{p}=3424$.

S Gupta, H Selvan, A Markan and V Gupta

Dr Rajendra Prasad Centre for Ophthalmic Sciences, All India Institute of Medical Sciences, New Delhi, India

Correspondence: S Gupta, Dr Rajendra Prasad Centre for Ophthalmic Sciences, All India Institute of Medical Sciences, New Delhi 110029, India Tel: +91 9910015999; Fax: +011 26588919. E-mail: dr.shikhagupta84@gmail.com 